\title{
Low Dissipative High Order Numerical Simulations of Supersonic Reactive Flows
}

\author{
B. Sjögreen ${ }^{1}$ and H.C. Yee ${ }^{2}$ \\ 1 NADA, KTH, 10044 Stockholm, Sweden (bjorns@nada.kth.se) \\ 2 NASA Ames Research Center, Moffett Field, CA 94035, USA (yee@nas.nasa.gov)
}

Motivation: In the modeling of viscous problems containing finite-rate chemistry, often, a wide range of space and time scales is present due to the reacting terms, over and above the different scales associated with turbulence flows, leading to additional numerical difficulties. This stems mainly from the fact that the majority of widely used numerical algorithms in reacting flows were originally designed to solve non-reacting fluid flows. It was shown in LeVeque \& Yee [1] that, for stiff reactions containing shock waves, it is possible to obtain stable solutions that look reasonable and yet are completely wrong, because the discontinuities are in the wrong locations. Due largely to numerical dissipations, stiff reaction waves move at nonphysical wave speeds, often at the rate of one grid cell per time step, regardless of their proper speed. There exist several methods that can overcome this difficulty for a single reaction term. For more than a single reacting term in fully coupled nonlinear systems, more research is needed. One impractical way of minimizing the wrong speed of propagation of discontinuities is to demand orders of magnitude grid size reduction compared with what appears to be a reasonable grid spacing in practice. It was also shown in Lafon \& Yee $[2,3]$ that the numerical phenomenon of incorrect propagation speeds of discontinuities may be linked to the existence of some stable spurious steady-state numerical solutions, and that the various ways in discretizing the reaction term can affect the stability of the overall scheme. Pointwise evaluation of the source terms appear to be the least stable. In addition, it was shown in Yee et al. and Griffiths et al. $[4,5]$ that spurious discrete traveling waves can exist, depending on the method of discretizing the source term. When physical diffusion is added, it is not known what type of numerical difficulties will surface.

Objective: The objective of this paper is to evaluate the performance of a newly developed low dissipative sixth-order spatial and 4th-order temporal scheme (Yee et al. and Sjögreen \& Yee [6-8]) for viscous reactive flows interacting with shock waves that contain fine scale flow structures. The accuracy and efficiency of the scheme, and to what degree the scheme can capture the correct physical wave speeds of stiff reactive flows will be included.

Numerical Methods: In the Yee et al. [6,7] method one time step consists of one step with a fourth-order or higher accurate non-dissipative spatial base scheme. Often an entropy split form of the inviscid flux derivative (Yee et al. Gerritsen \& Olsson $[7,9]$ ) is used along with a post processing step, where regions of oscillation are detected using an ACM (artificial compression method [10]) sensor, and filtered by adding the numerical dissipation portion of a shock capturing scheme at these parts of the solution. The entropy splitting of the inviscid flux derivative is considered as a conditioned form of the governing equations. The idea of the scheme is to have the spatially higher non-dissipative scheme activated at all times and to add the full strength, efficient and accurate numerical dissipation only at the shock layers. Thus, it is necessary to have good detectors which flag the layers, and not the oscillatory turbulent parts of the flow field. It was shown in Yee et al. [6,7] that the ACM sensor, while minimizing the use of numerical dissipation away from discontinuities, consists of tuning parameters and is physical problem dependent.

To minimize the tuning of parameters and physical problem dependence, new sensors with improved detection properties were proposed in Sjögreen \& Yee [8]. The new sensors are derived from utilizing appropriate non-orthogonal wavelet basis functions and they can be used to 
completely switch off the extra numerical dissipation outside shock layers. The non-dissipative spatial base scheme of arbitrarily high order of accuracy can be maintained without compromising its stability at all parts of the domain where the solution is smooth. Two types of redundant non-orthogonal wavelet basis functions are considered. One is the B-spline wavelet (Mallat \& Zhong [11] ) used by Gerritsen \& Olsson [9] in an adaptive mesh refinement method, to determine regions where refinement should be done. The other is the modification of the multiresolution method of Harten [12] by converting it to a new, redundant, non-orthogonal wavelet. The wavelet sensor is then obtained by computing the estimated Lipschitz exponent of a chosen physical quantity (or vector) to be sensed on a chosen wavelet basis function. Both wavelet sensors can be viewed as dual purpose adaptive methods leading to dynamic numerical dissipation control and improved grid adaptation indicators. Consequently, they are useful not only for shock-turbulence computations but also for chemical reaction and combustion simulations.

Numerical Example: For the numerical experiment, the same supersonic reactive flow problem as presented in Don \& Quillen and Don \& Gottlieb $[13,14]$ is used. The governing equations are the compressible Navier-Stokes equations with four species undergoing multi-chemical reactions. The chemical reaction is modeled by a single-step reversible reaction using $\mathrm{H}_{2}, \mathrm{O}_{2}, \mathrm{H}_{2} \mathrm{O}$ and $N_{2}$. A Prandtl number $P_{r}=0.72$, Schmidt number $S_{c}=0.22$ and the perfect gas equation of state approximation are used. The mixture specific heat at constant pressure were obtained from McBride et al. [15]. The Svehla [16] species viscosity constants and the Wilke's law model [17] for the mixture viscosity are used.

A two-dimensional planar shock in air interacting with hydrogen cylinder jets in three different initial configurations is considered. The three initial configurations are (a) a single jet, (b) two aligned jets, and (c) two non-aligned jets. The temperature of the hydrogen and air in the undisturbed region ahead of the shock is set to $1000^{\circ} \mathrm{K}$ with a pressure of $1 \mathrm{~atm}$. and zero velocity. The radius of the hydrogen cylinder is $1 \mathrm{~cm}$. A Mach 2 shock is placed at $x_{s}=0.5$ $\mathrm{cm}$. The computational domain is $0 \leq x \leq 0$. 175 and $-0.045 \leq y \leq 0.045$. Figure 1 shows snap shots of a single jet at six different stages of evolutionary process by the sixth-order ACM scheme $(\mathrm{ACM} 66)$ using a $500 \times 250$ grid. Figure 2 shows the comparison of the same scheme without the ACM sensor (TVD66) with ACM66 for a $500 \times 250$ and $1000 \times 500$ grids. Figure 2 shows a close-up of the region around the upper hydrogen bubble, at time $60 \mu \mathrm{s}$. There is an over all improvement in accuracy of the ACM66 over the TVD66 scheme, the fifth-order ENO $[13,14]$ and the Chebyshev collocation methods [13,14] for similar grids. The performance of WAV66 (same as ACM66 but with the sensor replaced by the wavelet sensor) is almost identical to that of ACM66, but requires no tuning of parameters.

Acknowledgment: The authors would like to thank Prof. W.S. Don for providing his spectral chemical reaction code as a guide to build our research code for the present study. 

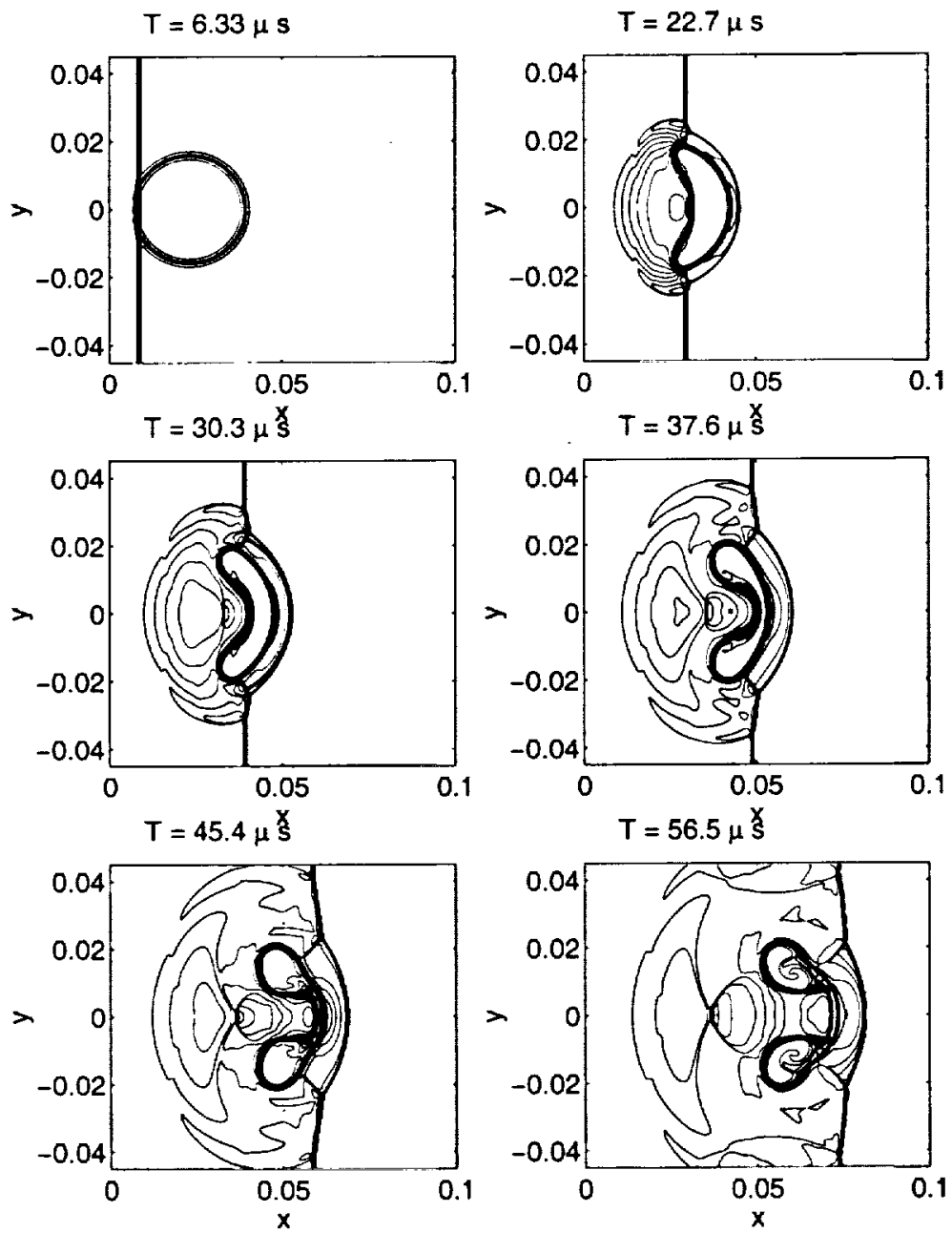

Fig. 1. Snap shots of density contours of the ACM66 simulation using a $500 \times 250$ grid.
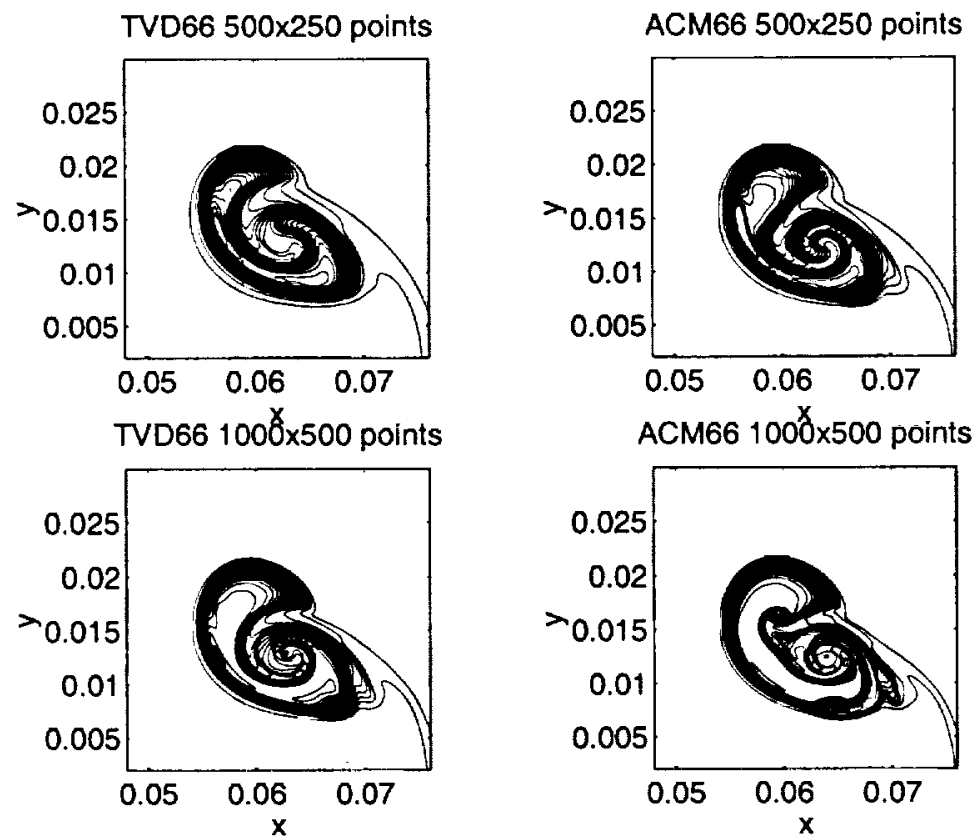

Fig. 2. Comparison between TVD66 and ACM66: $H_{2}$ mass fraction contours at $t=60 \mu s$ for a $500 \times 250$ and $1000 \times 500$ grids. 


\section{References}

[1] LeVeque, R.J. and Yee, H.C., "A Study of Numerical Methods for Hyperbolic Conservation Laws with Stiff Source Terms," J. Comput. Phys. 86 187-210 (1990).

[2] Lafon, A. and Yee, H.C., "Dynamical Approach Study of Spurious Steady-State Numerical Solutions of Nonlinear Differential Equations, Part IV: Stability vs. Numerical Treatment of Nonlinear Source Terms," Comp.. Fluid Dyn. 6 89-123 (1996).

[3] Lafon, A. and Yee, H.C., "Dynamical Approach Study of Spurious Steady-State Numerical Solutions for Nonlinear Differential Equations, Part III: The Effects of Nonlinear Source Terms in Reaction-Convection Equations," Comp.. Fluid Dyn. 6 1-36 (1996).

[4] Griffiths, D.F., Stuart, A.M. and Yee, H.C., "Numerical Wave Propagation in Hyperbolic Problems with Nonlinear Source Terms," SIAM J. of Numer. Analy. 29 1244-1260 (1992).

[5] Yee, H.C., Sweby, P.K. and Griffiths, D.F., "Dynamical Approach Study of Spurious Steady-State Numerical Solutions for Nonlinear Differential Equations, Part I: The Dynamics of Time Discretizations and Its Implications for Algorithm Development in Computational Fluid Dynamics," NASA TM-102820, April 1990; J. Comput. Phys. 97 249-310 (1991).

[6] Yee, H.C., Sandham, N.D., and Djomehri, M.J., "Low Dissipative High Order ShockCapturing Methods Using Characteristic-Based Filters," J. Comput. Phys. 150 199-238 (1999).

[7] Yee, H.C., Vinokur, M., and Djomehri, M.J., "Entropy Splitting and Numerical Dissipation," J. Comput. Phys. 162, 33-81 (2000).

[8] Sjögreen, B. and Yee, H.C., "Multiresolution Wavelet Based Adaptive Numerical Dissipation Control for Shock-Turbulence Computations," RIACS Technical Report 01.01 (2000).

[9] Gerritsen, M. and Olsson, P., "Designing an Efficient Solution Strategy for Fluid Flows," J. Comput. Phys. 129 245-262 (1996).

[10] Harten, A., "The Artificial Compression Method for Computation of Shocks and Contact Discontinuities: III. Self-Adjusting Hybrid Schemes," Math. Comp. 32 363-389 (1978).

[11] Mallat, S.G. and Zhong, S., "Characterization of Signals from Multiscale Edges," IEEE Transactions on Pattern Analysis and Machine Intelligence, 14 710-732 (1992).

[12] Harten, A., "Multiresolution Algorithms for the Numerical Solution of Hyperbolic Conservation Laws," Comm. Pure Appl. Math. 48 1305-1342 (1995).

[13] Don, W.S. and Quillen, C. B., "Numerical simulation of Shock-Cylinder Interactions," J. Comput. Phys. 122 244-265 (1995).

[14] Don, W.S. and Gottlieb, D., "Spectral Simulation of Supersonic Reactive Flows," SIAM J. Numer. Anal. 35 2370-2384 (1998).

[15] McBride, B.J., Heimel, S., Ehlers, J.G. and Gorden, S., "Thermodynamics Properties to $6000^{\circ} \mathrm{K}$ for 210 Substances Involving the first 18 elements," NASA SP-3002 (1963)

[16] Wilke, C.R., "A Viscosity Equation for Gas Mixtures," Chem. Phs. 18 517-519, (1950).

[17] Svehla, R.A., "Estimated Viscosities and Thermal Conductivities of Gases at High temperatures, NASA Report TR R-132 (1962). 